\title{
Microfluidic Platform For Sorting Materials Based On Their Densities Using Magnetic Levitation
}

\author{
Esra YILMAZ \\ Department of Bioengineering \\ Izmir Institute of Technology \\ Izmir,Turkey \\ esrayilmaz@iyte.edu.tr
}

\author{
Engin OZCIVICI \\ Department of Bioengineering \\ Izmir Institute of Technology \\ Izmir,Turkey \\ enginozcivici@iyte.edu.tr
}

\author{
H. Cumhur TEKIN \\ Department of Bioengineering \\ Izmir Institute of Technology \\ Izmir,Turkey \\ cumhurtekin@iyte.edu.tr
}

\begin{abstract}
Circulating Tumor Cells (CTCs) play a vital role in cancer diagnosis, prognosis and personalized medicine. However, CTCs are extremely rare in blood (i.e., down to 1-100 CTC per $1 \mathrm{~mL}$ human blood) and hard to isolate because of the heterogeneity of CTCs in biomarker expression. The current CTC separation and identification techniques use numerous differences between cells such as size, electric charges, density and expression of cell surface markers. However, these techniques have many limitations in terms of laborious sample preparation steps, inconsistent results caused by low specificity and efficiency and high cost. Hence, there is no standard method for isolating CTCs yet. With this study, it was aimed to fill the gap in CTC isolation and identification by proposing to develop a new method based on magnetic levitation principle, which has recently been demonstrated as a highly acceptable method for biological characterization of cells and monitoring of their cellular events. In this study, we have developed a new labelfree microfluidic sorter to separate microparticles/cells based on their densities using magnetic levitation principle. Two different density microparticles $(1.02 \mathrm{~g} / \mathrm{mL}$ and $1.09 \mathrm{~g} / \mathrm{mL})$ have been sorted and quantified in a continuous flow using a set of permanent magnets located in a 3D printed structure surrounding the microfluidic channel. This device can be used for rapid, low cost and label-free in-vitro diagnosis of cancer by sorting CTCs from whole blood in a highthroughput manner. The sorted cells might further be used for downstream analysis for personalized and precision medicine.
\end{abstract}

Keywords—magnetic levitation, microfluidics, densitybased measurement, cell sorting.

\section{INTRODUCTION}

Sorting of single or multiple target cells from a heterogeneous mixture is an important research area with implications on cancer diagnostics, cell therapeutics and stem cell research [1]. The commercial cell sorters such as fluorescence-activated cell sorting (FACS) and magnetic- activated cell sorting (MACS) rely on cell surface markers [2]. Although FACS and MACS are the most widely used methods, they have several limitations including laborintensive protocols and high processing costs $[1,3]$.

Cancer metastasis is the major cause (more than 90\%) of cancer-related death. Feasible tumor cells frequently change their genetic and biological forms that can invade blood vessels and migrate around the body as circulating tumor cells (CTCs). The separation/sorting techniques were classified by the induced cell properties by fluorescent and magnetic labels, size, shape, density, compressibility, deformability, cell surface markers, dielectric and intrinsic magnetic properties to distinguish cells. But there is no standard property or parameter is available to separate CTCs from blood cells. Hence, there is a great challenge in the detection and capture of CTCs because of the number of CTCs in the peripheral blood range from 1 to 100 cells per $\mathrm{mL}$ even biomarkers on the surface of the CTCs or inside the cells are not abundant.

Magnetic levitation through negative magnetophoresis is a novel and simple method to measure single-cell density [4-8]. Paramagnetic salt solutions and ferrofluids were frequently used to levitate objects to positions based on their densities to perform a measurement in non-flow systems. This type of separation is generally called as magnetic levitation, or briefly "MagLev" [4]. Gadolinium $\left(\mathrm{Gd}^{3+}\right)$ based nonionic paramagnetic medium was used to detection density changes at a single cell level without the need for any biomarker. This levitation platform, MagDense, is presented as an only platform that is sensitive small differences in density of cells at higher resolution $1 \times 10^{-4} \mathrm{~g} / \mathrm{mL}$ and allow them to monitor as real-time during the label-free assay [4]. By adding a sorting mechanism, the proposed technology will allow the diagnosis of cancer by sorting CTCs from whole blood. In addition, isolated CTCs with this novel technology can be used in transcriptomic and proteomic analyzes sensitively in personalized medicine.

Here, we introduce a new label-free microfluidic sorting platform based on magnetic levitation principle to 
isolate microparticles/target cells using their intrinsic density values.

\section{THEORY}

Our magnetic levitation platform is composed of two opposing magnets and a microfluidic channel in between these magnets. In the microfluidic channel, microparticles spiked in paramagnetic solution (i.e. gadolinium) tend to move towards the middle of the two magnets, where the magnetic field is minimum. Microparticles are levitated to a position where the applied magnetic force $\left(\mathrm{F}_{\mathrm{mag}}\right)$ is equal to the buoyancy force $\left(\mathrm{F}_{\mathrm{b}}\right)$ (Fig. 1). The levitation height of a microparticle is determined by magnetic susceptibility and density difference between the microparticle and paramagnetic solution [4]. Hence, microparticles are levitated to a distinct levitation height with respect to their densities. During levitation, these particles can be dragged in a microfluidic channel with an applied flow and they can be directed to the corresponding bottom/top outlet via a microfluidic separator. Hence, microparticles with distinct densities can be collected from these outlets. For instance, high density microparticles will be collected from the bottom outlets, while low density microparticles will be collected from the top outlet.

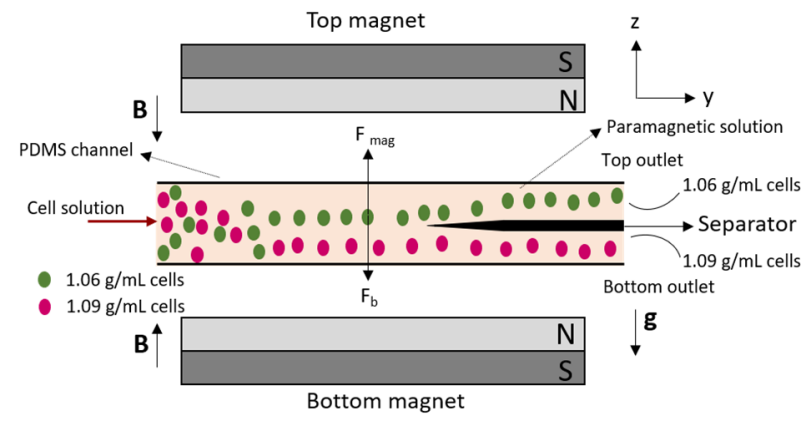

Fig. 1. The illustration of cell sorting based on the magnetic levitation principle. Cells reach an equilibrium levitation height where the magnetic force $\left(\mathrm{F}_{\mathrm{mag}}\right)$ equals the buoyancy force $\left(\mathrm{F}_{\mathrm{b}}\right) .1 .09 \mathrm{~g} / \mathrm{mL}$ cells are levitated close to the bottom magnet whereas 1.06 are levitated close to the top magnet. Hence, $1.06 \mathrm{~g} / \mathrm{mL}$ and $1.09 \mathrm{~g} / \mathrm{mL}$ cells are collected from top and bottom outlets, respectively. $\mathrm{g}$ and $\mathrm{B}$ specify gravitational acceleration and magnetic flux density, respectively.

Cancer cells $(<1.06 \mathrm{~g} / \mathrm{mL})$ show low density profiles compared to blood cells $(>1.09 \mathrm{~g} / \mathrm{mL})$ [4]. We simulated the trajectories of $1.06 \mathrm{~g} / \mathrm{mL}$ and $1.09 \mathrm{~g} / \mathrm{mL}$ cells having 20 $\mu \mathrm{m}$ diameter in the microfluidic channel under $10 \mu \mathrm{L} / \mathrm{min}$ flow using finite element modeling tools to show the applicability of our platform to sort CTCs from blood cells. The position of the separator in the microfluidic channel used in the sorting experiments corresponds to a levitation height of $\sim 200 \mu \mathrm{m}$ from the bottom of the channel. According to our results, $50 \mathrm{~mm}$ channel length is capable of directing $1.06 \mathrm{~g} / \mathrm{mL}$ and $1.09 \mathrm{~g} / \mathrm{mL}$ cells to the top and bottom outlets for collection, respectively (Fig. 2).

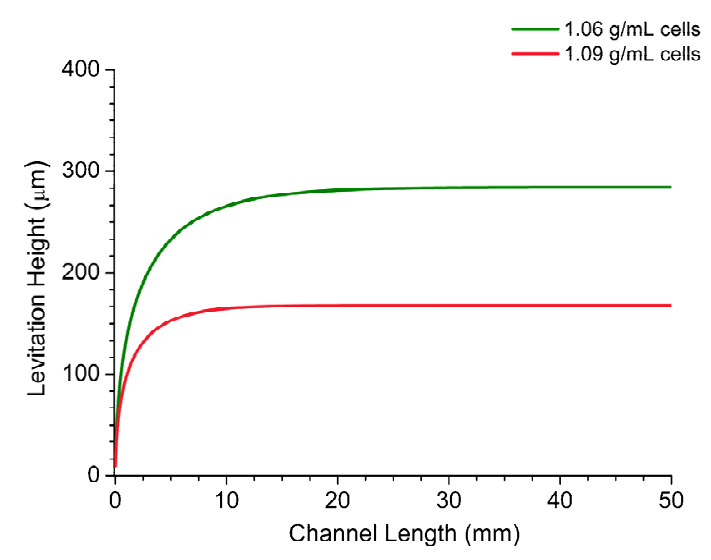

Fig. 2. Simulated trajectories of cells obtained from the finite element modeling tool along the channel length $(50 \mathrm{~mm})$ according to their densities. Levitation height is represented as the distance from the bottom of the channel. The microfluidic separator is located at $200 \mu \mathrm{m}$ height, which is $650 \mu \mathrm{m}$ away from the bottom magnet. In simulations, it is assumed that cells spiked in $30 \mathrm{mM}$ Gadavist solution are given from the $(0,0)$ coordinate (channel length, levitation height), which is one of the farthest point from the separator.

\section{EXPERIMENTAL}

\section{A. The Magnetic Levitation Platform}

The magnetic levitation platform is composed of three components: (i) two high-grade (N52) neodymium (NdFeB) magnets $(50 \mathrm{~mm}$ length, $2 \mathrm{~mm}$ width and $5 \mathrm{~mm}$ height) with same poles facing each other, (ii) a polydimethylsiloxane (PDMS) microfluidic chip in between the magnets, and (iii) four mirrors tilted $45^{\circ}$ for monitoring inside the microfluidic channel using an inverted microscope (Fig. 3). These components are assembled together with $3 \mathrm{D}$ printed parts. To do so, parts were designed and drawing files were saved in the appropriate format (with the extension.stl) to be printed using stereolithography 3D printer (Formlabs Form 2). Then, the necessary support parts were added to the design with the PreForm software and the parts were ready for printing. Afterward, final versions of the drawing files (with the extension. form) were sent to the $3 \mathrm{D}$ printer and printing was started. 3D printing process was carried out using "Clear v2 FLGPCL02" photoreactive resin with a resolution of $0.025 \mathrm{~mm}$.

After the printing process, printed parts were scraped off from the printer surface. According to the recommendation of the manufacturer, the printed parts were washed twice with isopropyl alcohol for 10 minutes. After treatment with isopropyl alcohol, the pieces were dried with filter paper and support parts were cut away. After the parts were ready, platform components are placed on them. 


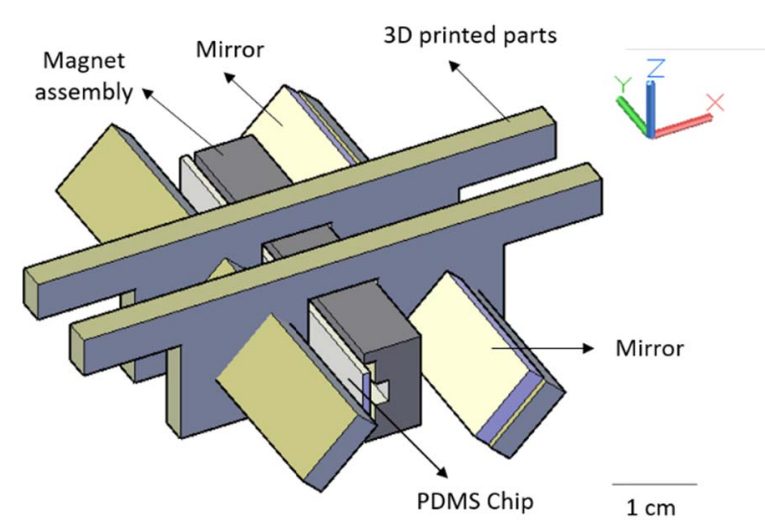

Fig. 3. A schematic drawing of the magnetic levitation platform. Magnets, mirrors and PDMS chip are reunited together via three dimensional (3D) printed parts.

\section{B. Fabrication of Microfluidic Chip}

PDMS chip with a single inlet and two outlets was fabricated using soft lithography technique [9]. 3D printed molds were required for the placement of the $\mathrm{Si}$ wafer with SU-8 patterns to form desired channel wall thicknesses. These 3D printed molds were fabricated by using the same fabrication procedure of magnetic levitation platform parts. After that, molds are assembled together with Si wafer with screws.

PDMS Sylgard 184 was used to fabricate the microfluidic chip. PDMS is a two-part polymer composed of silicone elastomer base and curing agent. PDMS with 10 (elastomer base): 1 (curing agent) ratio was prepared and mixed. The prepared PDMS was stored in the desiccator until the air bubbles inside PDMS were removed. Then, air bubble-free PDMS was slowly poured onto the mold assembly (Fig. 4). Then, the assembly was kept in the oven for 12 hours at $65{ }^{\circ} \mathrm{C}$ to cure PDMS. After that, PDMS chip was peeled from the mold. Inlets and outlets of the chip were drilled with a pointed needle having $1.4 \mathrm{~mm}$ inner core. PDMS chip was bonded on a glass slide surface with oxygen plasma treatment (Zepto Plasma, Diener) for $2 \mathrm{~min}$ at $100 \mathrm{~mW}$. Hence, the microfluidic chip with single inlet and two outlets was successfully produced (Fig. 5).

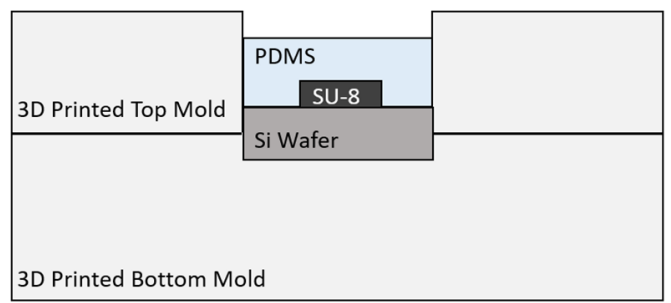

Fig. 4. The cross-sectional view of the mold to fabricate the microfluidic chip.

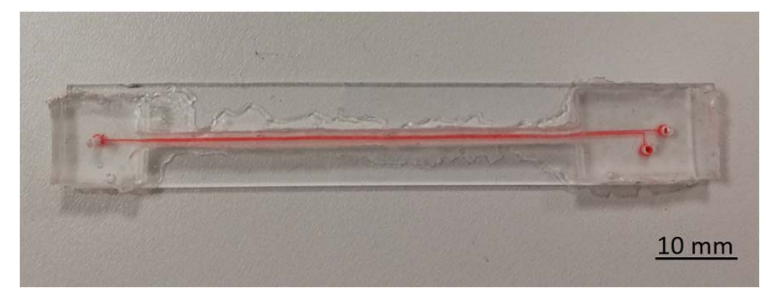

Fig. 5. Fabricated microfluidic chip with single inlet (left) and two outlets (right). Microfluidic channels were filled with red food color solution.

\section{Sorting Setup}

The fabricated microfluidic chip was mounted the magnetic levitation platform. Flow inside the microfluidic channel was controlled using a syringe pump (NE-1600 New Era Pump Systems) equipped with a $1 \mathrm{~mL}$ syringe. The syringe was attached to the chip inlet with flexible tubing (TYGON, Cole Parmer). After the magnetic levitation platform was placed on an inverted microscope (ZEISS Axio Vert A1), microfluidic channel was monitored using the $5 \times$ objective via tilted mirrors on the magnetic levitation platform. The sorting setup was shown in Fig. 6.

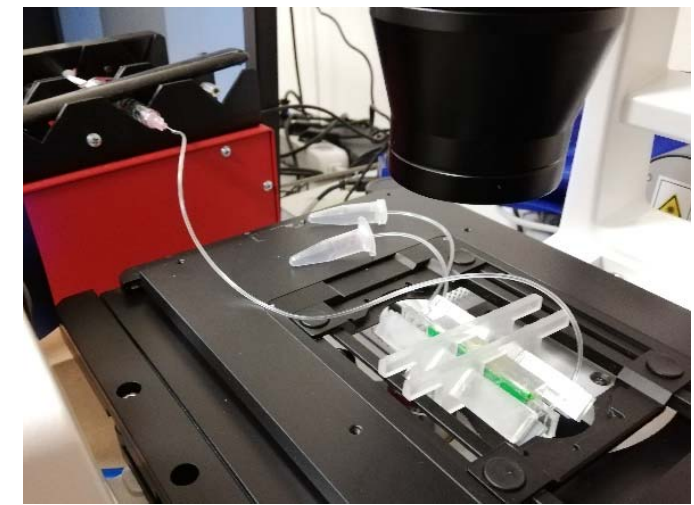

Fig. 6. The sorting setup consisting of the magnetic levitation platform the syringe pump and the microscope.

\section{Sorting Experiments}

For sorting experiments, polyethylene microparticles with different densities $(1.02 \mathrm{~g} / \mathrm{mL}$ and $1.09 \mathrm{~g} / \mathrm{mL})$ were used. These microparticles with $10-27 \mu \mathrm{m}$ size were purchased from Cospheric LLC. The particles were prepared in fetal bovine serum containing $30 \mathrm{mM}$ Gadavist [7]. The prepared solution having 10000 microparticles/mL concentration was loaded into the microfluidic channel using a syringe pump at $10 \mu \mathrm{L} / \mathrm{min}$ flow rate. The sorting of the microparticles was observed from the separator region within the microfluidic channel.

\section{E. Measuring Densities of Microparticles}

Magnetic properties of microparticles are lower than the paramagnetic solution used to spike microparticles. 
Hence, particles are levitated by the magnetic forces and move towards the middle of the two magnets where the magnetic field is minimum. At the levitation position where the magnetic force is equal to the buoyancy force, microparticles remain stationary. The levitation position depends on the density of microparticles. Thus, by looking at the levitation height of the microparticles, the density of microparticles can be determined.

Microparticles densities were also measured in the magnetic levitation platform [10]. To do so, a glass microcapillary channel $(1 \mathrm{~mm} \times 1 \mathrm{~mm} \times 50 \mathrm{~mm})$ was used instead of a microfluidic channel. Microparticles are levitated in $30 \mathrm{mM}$ Gadavist solution for $10 \mathrm{~min}$. Afterward, their levitation heights were measured and translated to density values.

\section{RESULT AND DISCUSSION}

The sorting efficiency of the platform was evaluated with two types of microparticles having different densities (1.02 and $1.09 \mathrm{~g} / \mathrm{mL}$ ). As shown in Fig. 7, most of the 1.02 $\mathrm{g} / \mathrm{mL}$ microparticles were directed to the top outlet whereas most of the $1.09 \mathrm{~g} / \mathrm{mL}$ microparticles were directed to the bottom outlet. Sorting efficient was also analyzed by counting the number of microparticles collected from the outlets. With proposed strategy, $87.5 \%$ of $1.02 \mathrm{~g} / \mathrm{mL}$ microparticles were collected from the top outlet and only $5.32 \%$ of $1.09 \mathrm{~g} / \mathrm{mL}$ microparticles sorted through top outlet while using a $10 \mu \mathrm{L} / \mathrm{min}$ flow rate (Fig. 8). At the same time, $7.5 \%$ of $1.02 \mathrm{~g} / \mathrm{mL}$ microparticles were collected from the bottom outlet under $10 \mu \mathrm{L} / \mathrm{min}$ flow rate.

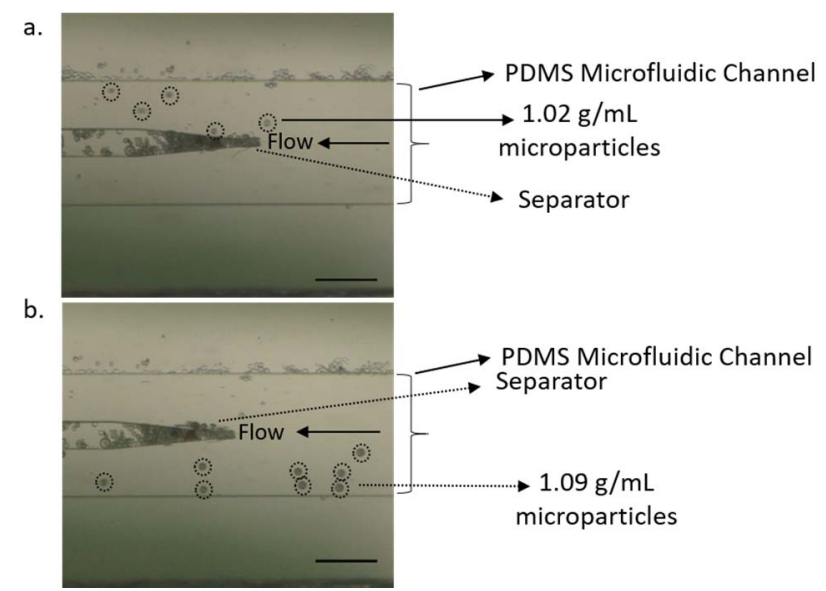

Fig. 7. Micrographs of (a) $1.02 \mathrm{~g} / \mathrm{mL}$ (b) $1.09 \mathrm{~g} / \mathrm{mL}$ microparticles at the channel outlets. Scale bars indicate $200 \mu \mathrm{m}$.

Density distribution of microparticles were also analyzed in the magnetic levitation platform by measuring levitation heights of microparticles (Fig. 9). $1.02 \mathrm{~g} / \mathrm{mL}$ and $1.09 \mathrm{~g} / \mathrm{mL}$ particles showed $0.95-1.10 \mathrm{~g} / \mathrm{mL}$ and 0.97 $1.41 \mathrm{~g} / \mathrm{mL}$ density distribution. Since these distributions are overlapping densities, it is expected that some of 1.02 $\mathrm{g} / \mathrm{mL}$ microparticles can be collected from the bottom outlet and some of the $1.09 \mathrm{~g} / \mathrm{mL}$ microparticles can be collected from the top outlet as also observed in the experiments (Fig. 8). Nevertheless, our platform shows high sorting efficiency $(\sim 90 \%)$ with a good throughput $(\sim 1 \mathrm{~mL} / \mathrm{h})$.

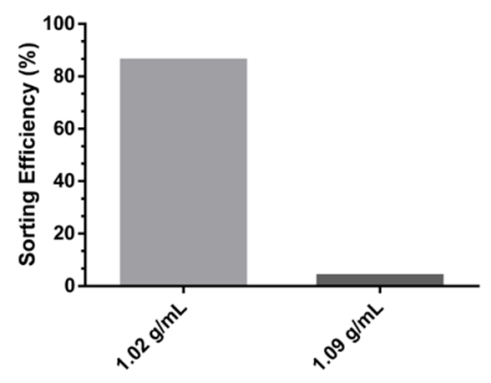

Fig. 8. Sorting efficiency of microparticles from the top outlet.

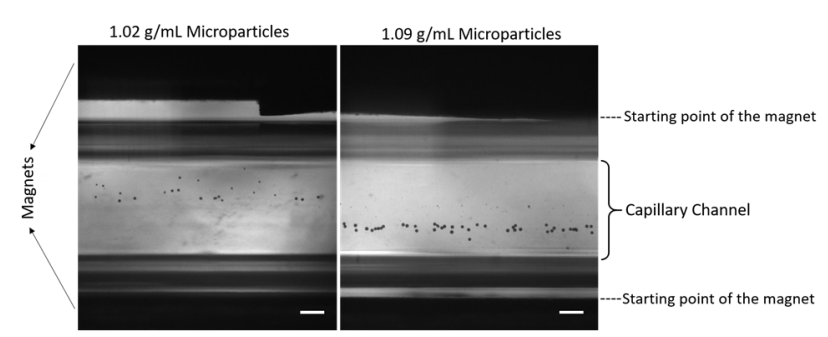

Fig. 9. Microscopic images of the $1.02 \mathrm{~g} / \mathrm{mL}$ and $1.09 \mathrm{~g} / \mathrm{mL}$ density particles in solutions containing $30 \mathrm{mM}$ on the magnetic levitation platform. The scale bars show $200 \mu \mathrm{m}$.

We showed for the first time sorting of the different density of microparticles using magnetic levitation strategy in a microfluidic chip. As future work, sorting performance will be evaluated for different cell lines (e.g., cancer cells and blood cells) in different cell concentrations. Since cancer cells show lower density profiles compared to blood cells, we believe that our platform would be capable of sorting and identifying cancer cells from blood samples. Hence, this platform would be useful for label-free CTC analysis by using density as a biomarker. Sorted CTCs would be utilized for rapid and low-cost diagnosis and prognosis of cancer.

\section{V.CONCLUSION}

As a result of the study, in the developed magnetic levitation platform, the different density of microparticles have been sorted with high sorting efficiency ( $\sim 90 \%$ sorting efficiency from top outlet for $1.02 \mathrm{~g} / \mathrm{mL}$ particles and $\sim 95 \%$ sorting efficiency from bottom outlet for 1.09 $\mathrm{g} / \mathrm{mL}$ particles) for the first time on the microfluidic chip using magnetic levitation principle. The magnetic levitation principle has the potential to overcome all limitations including labor-intensive protocols and high processing costs by detecting a small number of CTCs within the blood cells. The platform-based magnetic 
levitation strategy can be presented as a novel, reliable, simple, low cost and less risky procedure for the early diagnosis of cancer compared to existing clinical methods.

\section{ACKNOWLEDGMENT}

Financial support by The Scientific and Technological Research Council of Turkey for 116M298 grant is gratefully acknowledged.

\section{REFERENCES}

[1] S. Yaman, M. Anil-Inevi, E. Ozcivici, H.C. Tekin, "Magnetic Force-Based Microfluidic Techniques for Cellular and Tissue Bioengineering", Front. Bioeng. Biotechnol., vol. 6, no. 192, December 2018.

[2] M. Zborowski and J.J. Chalmers, "Rare cell separation and analysis by magnetic sorting", Anal. Chem., vol. 83, no. 21, pp. 8050-8056, August 2011.

[3] C.W. Shields, C.D. Reyes and G.P. Lopez, "Microfluidic cell sorting: a review of the advances in the separation of cells from debulking to rare cell isolation", Lab. Chip, vol. 15, no. 5, pp 1230-1249, February 2015.

[4] N.G. Durmus, H.C. Tekin, S. Guven, K. Sridhar, A. Arslan-Yildiz, G. Calibasi, I. Ghiran, R.W. Davis, L.M. Steinmetz, U. Demirci, "Magnetic levitation of single cells", Proc. Natl. Acad. Sci. USA, vol. 112, no. 28, pp. E3661-3668, July 2015.

[5] O. Sarigil, M. Anil-Inevi, E. Yilmaz, G. Mese, H.C. Tekin, E. Ozcivici, "Label-free density-based detection of adipocytes of bone marrow origin using magnetic levitation", Analyst, vol. 144, no. 9, pp. 2942-2953, April 2019.

[6] S. Tasoglu, J.A. Khoory, H.C. Tekin, C. Thomas, A.E. Karnoub, I.C. Ghiran, U. Demirci, "Levitational Image Cytometry with Temporal Resolution", Adv. Mater., vol 27, no. 26, pp. 3901-3908, June 2015.

[7] M. Anil-Inevi, E. Yilmaz, O. Sarigil, H.C. Tekin, E. Ozcivici, "Single Cell Densitometry and Weightlessness Culture of Mesenchymal Stem Cells Using Magnetic Levitation", Methods Mol. Biol., 2019, pp.1-11.

[8] M. Anil-Inevi, S. Yaman, A.A. Yildiz, G. Mese, O. Yalcin-Ozuysal, H.C. Tekin, E. Ozcivici, "Biofabrication of in situ Self Assembled 3D Cell Cultures in a Weightlessness Environment Generated using Magnetic Levitation", Sci. Rep.,vol. 8, no. 1, pp. 7239-7248. May 2018.

[9] H.C. Tekin, M. Cornaglia, M.A. Gijs, "Attomolar protein detection using a magnetic bead surface coverage assay", Lab. Chip, vol. 13, no. 6, pp. 1053-1059, January 2013.

[10] H.C. Tekin, "Determining densities of micro-particles in a magnetic levitation platform using automated digital image analysis", in $201826^{\text {th }}$ IEEE Signal Processing and Communication Applications Conference; Izmir, Turkey, pp. 1-4, 2-5 May 2018. 